Critical Gambling Studies (2021)

Vol. 2, No. 1

Early Career Research

\title{
EDITORIAL \\ Early Career Research in the Gambling Field: A Reflection on Existing Challenges and Opportunities for Change
}

\author{
Eva Monson, Jennifer Reynolds
}

\begin{abstract}
We are incredibly proud to present this special issue on early career research for Critical Gambling Studies. As two early career researchers (ECRs) in the field, leading this project has been an honour and an incredibly rewarding experience. A first of its kind, we believe this special issue is an important contribution to gambling literature. With this in mind, the views expressed in this editorial are our own and are by no means meant to be representative of all ECRs in the gambling field. However, our perspectives have not only been shaped by personal experience but also reflect years of conversation with our peers, many of whom feel conflicted, overlooked, expendable, and isolated (now more than ever), and who are reaching their breaking point.
\end{abstract}

\section{The Broader Context to ECR Challenges}

Across all fields, ECRs struggle. Indeed, they show a higher level of emotional exhaustion than their more senior colleagues (Gonzalez \& Bernard, 2006; Watts \& Robertson, 2011). Constant expectation of high productivity and performance (e.g., publish or perish culture) coupled with low occupational job security for both research and teaching greatly contribute to early career burnout and excessive attrition of talented ECRs (Salimzadeh et al., 2017; Watts \& Robertson, 2011). Moreover, Nir \& Zilberstein-Levy (2006) argue that these pressures can lead to research that lacks originality and to a decreased willingness to use innovative research methods.

\section{ECRs in Gambling Studies}

The field of gambling studies is not immune to these problems. Indeed, since being appointed as guest editors for this special issue, one of us has left the gambling field to secure more stable employment. Situations like this are, unfortunately, all too common, with ECRs leaving the field for a variety of reasons. As in many fields, resources and opportunities are spread thin, but early career gambling scholars struggle with additional complexities, such as securing ethically sourced and sustainable funding, and navigating a field rife with fierce and sometimes unpleasant divisions (e.g., disputes about the best way to measure gamblingrelated problems). Early career scholars must also survive in an academic field where gambling operators have significant and sometimes direct influence over funding decisions and often control access to data and venues for data collection. The role of industry has resulted in claims that the field of gambling studies has become too 'safe': rewarding conformity and marginalizing critical voices (Cassidy et al., 2013; Cassidy, 2014; Young \& Markham, 2015). This reality is 'felt most acutely by early career researchers' (Cassidy et al., 2013, p. 346).

With this in mind, we would be remiss not to specifically acknowledge a major issue within the field concerning problematic power dynamics that, as recent events have demonstrated (Nicoll \& Akçayır, 2020), have yet to be duly addressed. Success and career trajectory-from getting a job, to funding, to publishing-are often shaped, if not dictated, by a select few senior members of the community, which leaves ECRs vulnerable to problematic and potentially predatory relationships. As ECRs in this field, we could easily add some of our experiences to the existing examples of egregious power abuses in gambling studies. Unfortunately, this editorial will not further rock the boat because, in order to speak truth to power in this situation, we would knowingly be putting ourselves at risk, given the precarity of the positions we hold and our status within the field. Indeed, the hierarchical nature of academia, amplified by the insular and politically charged context of the gambling field, make it almost impossible to be critical without some form of repercussion. Since we do not feel safe or comfortable writing about our specific experiences, we will focus on what can be done to create a better future for the field by highlighting opportunities to support and promote ECRs. We offer these thoughts not as a critique of 
specific people or organizations, but rather as a call to action for the field as a whole.

\section{A Call to Action}

In their recent blog post for Critical Gambling Studies, Nicoll and Akçayır (2020) proposed specific strategies to disrupt existing 'hierarchical systems' that have proven problematic. Their strategies include moving away from problematic, hierarchically organized, group research projects that privilege already powerful voices; cultivating space for soleauthored publications, as well as genuine interdisciplinary collaboration; and supporting 'more independent fora for early career researchers.' We would like to build on these ideas and provide specific, tangible suggestions that, if implemented, could greatly improve existing power dynamics: (1) Promotion of and advocacy for ECRs and their work; (2) Mentorship; and (3) Creating dedicated spaces for ECR collaboration and community building.

\section{Promotion and Advocacy}

While change within the field might have to be primarily driven by ECRs, it will be greatly accelerated with the support of more established researchers. Recognition of the existing power imbalance is essential, and senior researchers can advocate alongside ECRs by speaking out and pushing back against the status quo to improve conditions for the next generation of gambling scholars.

Researchers in more stable positions within the field (e.g., tenured professors) have an opportunity to support, uplift, and make space for ECRs. Beyond reading, disseminating, and citing their work, and inviting them to present as keynote speakers and expert panelists, promotion can mean involving them in grant review committees as well as editorial and conference boards. Including ECRs in these positions of relative prestige and power not only provides them with insights into how decisions are made, but it also demonstrates respect for their expertise. Ensuring representation also normalizes their participation in the field's decision-making process. Publishing is imperative for ECRs and editorial leadership positions are incredibly rare and that is why opportunities like this special issue put forth by Critical Gambling Studies are invaluable moving forward.

\section{Mentorship}

Good mentors, who are mindful of power dynamics and who find ways to subvert the hierarchical nature of academic relationships, make an incredible difference in the lives of their mentees. We have both been incredibly privileged to have mentors who were conscious of the legacy that they leave behind; not only in terms of research output, but in terms of what they have done to nurture the next generation of emerging scholars. They have gone out of their way to create spaces where ECRs feel secure and valued, and, as a result, have been able to develop critical thinking capacities and take risks that might not otherwise be possible. Mentors have fought on our behalf when the field was unjust and helped us navigate the politically charged minefield that is gambling studies. Some mentors have even shared their own varied experiences and struggles; this level of transparency both humanizes them and has allowed us to learn from their past mistakes. In the long run, we feel this type of mentorship will lead to more epistemological and methodological diversity, and cultivate openness to new paradigms and approaches to gambling research.

\section{Creating Dedicated Spaces for ECRs}

Nicoll and Akçayır's (2020) blog demonstrated evidence that: 'success in gambling studies requires very strong networks of collaborators.' In 2017, through multiple conversations with ECRs, it became apparent to us that, while there existed many traditional avenues to network, such as attending conferences and through mentors, there lacked specific opportunities for ECRs to build collaborative relationships with their peers. In response, we created the Research And Networking for Gambling Early-career Scholars (RANGES) community with the aim of fostering the interaction, capacity, and growth of early career scholars in gambling studies. Created by ECRs for ECRs, RANGES aims to bring together individuals from different disciplines (e.g., gambling/gaming studies), conceptual/ methodological backgrounds, and institutions to promote innovative methodological approaches and to adopt a broad and interdisciplinary vision for the future of gambling studies.

As a part of the RANGES mandate, we secured funding for and organized an inaugural international conference that took place in September 2019 at the Université de Sherbrooke in Quebec, Canada. The conference was a success. It hosted over 60 participants from six countries (Australia, Canada, France, Germany, New Zealand, and United Kingdom). One of the major achievements of our conference was the deliberate rejection of typical power dynamics that are so often embedded within traditional academic conferences, including the creation of dedicated spaces for ECRs to openly express themselves among their peers. This allowed for a level of openness in our discussions that we think surprised us all, and it facilitated the creation of lasting, genuine, high-quality collaborative relationships. More information about the organization of this conference and its specific goal of creating dedicated spaces for ECRs can be found in a forthcoming blog post for Critical Gambling Studies ${ }^{1}$.

\footnotetext{
${ }^{1}$ Critical Gambling Studies Blogs can be accessed here: https://criticalgamblingstudies.blogspot.com/
} 
Integrated within the planning of the RANGES conference was a call for papers by ECRs in the gambling field. Those papers now make up this special issue. Furthermore, as a direct result of discussions that took place at our conference, others have developed additional ECR networks. The growth of these types of networks will greatly increase opportunities for lateral collaboration among ECRs, which is essential for the future of gambling studies.

\section{Conclusion}

In view of the issues identified above, this editorial and special issue signify a step in the right direction for the broader field of gambling studies. If these suggestions are acted upon, we can create a better future for the next generation of ECRs and potentially slow the exodus of talented researchers from the gambling field. Yet, while we have highlighted important actions we can take at the individual level, we understand that there remain systemic issues that will require a broader commitment to change from the field as a whole.

\section{Article Summaries}

The articles in this special issue of Critical Gambling Studies clearly point to a diverse set of topics and crosscutting themes of importance to ECRs in the gambling field. The definition of 'critical' was broadened for the purposes of inclusivity, and to showcase and promote a variety of ECR work, epistemologies, and methodologies.

The first paper in this special issue, 'A Critical Analysis of Interventions for Women Harmed by Others' Gambling' (Palmer du Preez et al.), examines the underrepresented topic of family members and others who are affected by gambling harm. Using a feminist post-structuralist lens, the study provides insight into how women family members and affected others position themselves and their support needs in relation to gambling harm and recovery. Findings highlight how powerful constructs, practices, and implied values alienate women from gambling support services. The article argues that women and families affected by gambling harm require support that includes advocacy, community development, and services that take a more client-led and gender-aware approach.

'The Musings of "Evil Bastards": Perspectives from Social Casino Game Professionals' (Reynolds) examines a dimension of social casino games that has been largely unexplored in the existing literature: the experiences of the game professionals who design and develop the games. The convergence between gambling and gaming has important implications, not only for players, but also for the professionals who create these products. The results highlight a very real ethical struggle felt by social game professionals as a result of the dark design patterns underlying these games. The author shows the need for future research to examine how game design education addresses the convergence of gambling and gaming.

'Patterns of Disciplinary Involvement and Academic Collaboration in Gambling Research: A Co-Citation Analysis' (Akçayır et al.) uses bibliometric methods to examine a selection of gambling-related publications during the last five years. Mapping out unique citation patterns relating to disciplinary concentrations and academic collaborations, the authors found key clusters among the studies analyzed. Patterns reveal that gambling researchers primarily cited authors from the disciplines of neuroscience, psychology, health science, and psychiatry, with fewer citations from social sciences or humanities. Given that the findings indicate that gambling research is dominated by a medical and psychological focus on problem and pathological gambling, the authors highlight the need for greater collaboration between scholars in underrepresented disciplines, while also focusing on broader political and social contexts that influence gambling behaviour and regulation.

In 'General and Gambling-Specific Types of Control: Extending Mental Health Theory and Concepts to Problem Gambling,' Stark boldly expands our understanding of control and loss of control. Through in-depth interviews, this study highlights how different understandings of control, drawn from mental health research, can interact with and influence gamblingspecific beliefs. Findings illustrate the heterogeneous ways that gamblers subjectively experience and attribute meaning to control in their lives, thereby increasing our understanding of gambling problems.

In 'Health Promotion Strategies to Address Gambling-Related Harm in Indigenous communities: A Review of Reviews,' Whitty et al. present a systematic review of reviews of health promotion strategies relevant and applicable to addressing gambling-related harm in Indigenous communities. This timely review draws our attention to the importance of improving the health and well-being of Indigenous communities when addressing gambling-related harms. Findings illustrate the challenges of developing appropriate gambling-related health promotion strategies with, and for, Indigenous communities.

The paper by Fu et al., 'The Relationship Between Unexpected Outcomes and Lottery Gambling Rates in a Large Canadian Metropolitan Area,' is a refreshing take on the relationships between external contingencies and gambling behaviour. Exemplifying the varied methodologies and datasets that ECRs are using in their work, Fu et al. examine the daily fluctuations in lottery sales in Toronto, Canada, as a function of 'prediction errors' for weather (i.e., sunshine) and sports outcomes. The results partially replicate findings from similar datasets in the United States, but also demonstrate the malleability of lottery gambling behaviours in response to incidental events between geographies and cultures. 
'Gender Equality in Gambling Student Funding: A Brief Report' by Leonard and Violo tackles the important issue of gender disparity in academia. Drawing from the Alberta Gambling Research Institute's (AGRI) master'sand doctoral-level scholarship recipients, the authors sought to explore gender equality in graduate-level scholarship award distribution. Findings illustrate that, from 2009-2019, AGRI distributed graduate-level scholarships equally across genders. In line with this special issue, the authors suggest that future research should examine the career trajectories of these award recipients.

Hahmann \& Monson's article, 'Rationalization as a Dissonance Management Strategy among Electronic Gambling Machine Players,' provides an analysis of gambling behaviour that goes beyond conventional, cognitive behavioural paradigms by centring the internal logic that informs and shapes the behaviour of electronic gambling machine (EGM) players. Using a theoretical model anchored in the social scientific study of religious responses to prophetic disconfirmations, the authors find that EGM players use a similar approach to dissonance management as members of religious groups. Their qualitative findings offer key insight into the complexity of dissonance management strategies that could be of benefit to gambling researchers, and prevention and treatment specialists. While cognitive behavioural therapy is the foremost therapeutic approach used to treat problem gambling, more holistic approaches might warrant consideration in light of findings here that emphasize the significance of spiritual and religious beliefs about morality, God, and supernatural forces.

Jääskeläinen et al.'s study examines residents' perspectives on a new urban casino in Tampere, Finland, in 'Ambiguity and Abjection: Residents' Reactions to a New Urban Casino.' Using qualitative focus groups with residents prior to the opening of a new casino, findings reveal several incompatibilities between casino gambling and the daily lives of the locals who reside in Tampere. Four key dimensions were identified: residents' self-understanding; the contract between the municipality and local residents; Tampere's city image; and the evaluation of the pros and cons. The authors conclude that gambling locations should take into consideration relevant cultural-spatial contexts, including those of the local residents whose daily lives will be impacted.

Our final paper, 'A Genealogical Analysis of the Medical Model of Problem Gambling' (Wilcox), calls attention to the powerful effect of framing problem gambling through the lens of the medical model on gamblers' subjectivity and their relationship to gambling. This historically rich critical investigation applies Foucault's genealogical method to reveal the cumulative effect of medical framings on people's sense of self.

Finally, this special issue includes two reviews of Rebecca Cassidy's latest book, Vicious Games:
Capitalism and Gambling (2020). In keeping with the theme of this special issue, we chose to ask two researchers, Annie-Claude Savard and Garry Smith, who are at different stages in their careers (early career and senior researcher, respectively) to write parallel reviews of Vicious Games in the hopes of cultivating different perspectives on this interesting and highly anticipated book.

\section{References}

Cassidy, R. (2014). Fair game? Producing and publishing gambling research. International Gambling Studies, 14(3), 345-353. https://doi.org/10.1080/14459795.2014.971420

Cassidy, R., Loussouarn, C., \& Pisac, A. (2013). Fair game: Producing gambling research. The Goldsmiths Report. Retrieved from https://www.gold.ac.uk/gamblingineurope/report

Gonzalez, S., \& Bernard, H. (2006). Academic workload typologies and burnout among faculty in Seventh-day Adventist colleges and universities in North America. Journal of Research on Christian Education, 15(1), 13-37. https://doi.org/10.1080/10656210609484992

Nicoll, F., \& Akçayır M. (2020). A response to gambling studies' \#MeToo moment. Critical Gambling Studies Blog. https://doi.org/10.29173/cgs63

Nir, A. E., \& Zilberstein-Levy, R. (2006). Planning for academic excellence: Tenure and professional considerations. Studies in Higher Education, 31(5), 537-554. https://doi.org/10.1080/03075070600922725

Salimzadeh, R., Saroyan, A., \& Hall, N. C. (2017). Examining the factors impacting academics' psychological well-being: A review of research. International Education Research, 5(1), 13-44. https://doi.org/10.12735/ier.v5n1p13

Watts, J., \& Robertson, N. (2011). Burnout in university teaching staff: A systematic literature review. Educational Research, 53(1), 3350. https://doi.org/10.1080/00131881.2011.552235

Young, M., \& Markham, F. (2015). Beyond disclosure: Gambling research, political economy, and incremental reform. International Gambling Studies, 15(1), 6-9. https://doi.org/10.1080/14459795.2014.995201

\section{Acknowledgements}

As guest editors of this special issue, we have many people we need to thank. First and foremost, we would like to thank all of the early career researchers who contributed as authors to this special issue. We are incredibly grateful to Fiona Nicoll for supporting our interest in developing a special issue focused exclusively on highlighting research conducted by early career scholars in gambling studies-the first of its kind. Thank you for creating a platform that makes this special issue possible and allowing our idea to blossom into what it is today. We must also thank Kate Bedford who guided us through our first editorial experience with an unlimited amount of patience. During an incredibly trying time in all of our lives, Kate encouraged us to see this project through and gave an untold number of hours to make sure that it would be of the utmost quality. She was an incredible sounding board throughout this process. We would like to thank all of the reviewers of this collection of papers for their insightful, supportive, and timely reviews. A very special thank you to Elise Sammons, who provided technical 
support and copyediting during the final weeks of this process. We would also like to thank Jonathan Leggo for his copyediting work, and Nicole Arsenault for her feedback on the ideas included in this work. We would like to thank everyone involved for their patience throughout the unexpected delays in the publishing process, an inevitable result of this incredibly difficult year.

\section{Funding Statement}

This project was generously funded by a SSHRC Connection grant (611-2018-0385).

Monson currently receives salary support from the Fonds de recherche du Québec-Société et culture (FRQSC). In the past three years, she has additionally received funding as a principal investigator from $F R Q-$ SC and the Social Sciences and Humanities Research Council (SSHRC), as well as a co-investigator funding from FRQSC and the Canadian Institutes of Health Research (CIHR).

Jennifer Reynolds received postdoctoral funding via an Action-Concertée grant from Quebec's Fonds de Recherche-Société et Culture (FRQ-SC; 210145).

\section{Author Details}

Eva Monson is a professor at Université de Sherbrooke (Canada) and a researcher at the Centre de recherche Charles-Le Moyne-Saguenay-Lac-SaintJean sur les innovations en santé (CR-CSIS). Her current research is devoted to investigating how social and environmental deprivation, from the level of the individual to the neighbourhood in which they reside, influence gambling practices.

Jennifer Reynolds is an applied researcher and knowledge broker. Trained in Public Health at the University of Toronto, she has over 15 years of research experience on the topic of gambling problem prevention, with an expertise in youth. Reynolds codeveloped the first set of best practices for youth gambling problem prevention and has produced two documentaries, along with many other arts-based initiatives as gambling prevention/education resources. Her current interests focus on using participatory action approaches to engage young people in arts-based knowledge mobilization. 